\title{
IN-LINE CHEMICAL SENSOR DEPLOYMENT IN A TRITIUM PLANT
}

\author{
$\underline{\text { J. S. Wright }}{ }^{a}$, R. D. Torres $\underline{\underline{b}}$, B. Peters $\underline{\underline{b}}$, D. T. Hope $e^{\underline{a}}$, and $\underline{\text { L. L. Tovo }}$ To $^{b^{*}}$ \\ ${ }^{a}$ Savannah River Nuclear Solutions, Aiken, SC, USA; *laura.tovo@srnl.doe.gov \\ ${ }^{b}$ Savannah River National Laboratory, Savannah River Site, Aiken, SC, USA
}

The Savannah River Tritium Plant (TP) relies on well understood but aging sensor technology for process gas analysis. Though new sensor technologies have been brought to various readiness levels, the TP has been reluctant to install technologies that have not been tested in tritium service. This gap between sensor technology development and incorporating new technologies into practical applications demonstrates fundamental challenges that exist when transitioning from status quo to state-of-the-art in an extreme environment such as a tritium plant. These challenges stem from three root obstacles: 1) The need for a comprehensive assessment of process sensing needs and requirements; 2) The lack of a pick-list of process-compatible sensor technologies; and 3) The need to test technologies in a tritium-contaminated process environment without risking production.

At Savannah River, these issues are being addressed in a two phase project. In the first phase, TP sensing requirements were determined by a team of process experts. Meanwhile, Savannah River National Laboratory sensor experts identified candidate technologies and related them to the TP processing requirements. The resulting roadmap links the candidate technologies to actual plant needs. To provide accurate assessments of how a candidate sensor technology would perform in a contaminated process environment, an instrument demonstration station was established within a TP glove box. This station was fabricated to TP process requirements and designed to handle high activity samples. The combination of roadmap and demonstration station provides the following assets:

- Creates a partnership between the process engineers and researchers for sensor selection, maturation, and insertion,

- $\quad$ Selects the right sensors for process conditions

- Provides a means for safely inserting new sensor technology into the process without risking production, and

- Provides a means to evaluate off normal occurrences where and when they occur.

This paper discusses the process to identify and demonstrate new sensor technologies for the Savannah River TP.

\section{INTRODUCTION}

Global budget reductions and continuing worldwide austerity measures are pressuring tritium fuel cycle processes, including those at the Savannah River Site (SRS), to be more efficient, cost effective, and to be more responsive to changing delivery demands, while maintaining delivery of high quality material(s) on time. At the same time, growing global demand for tritium in fusion energy interests like ITER and the National Ignition Facility, and for radiological medical fields have pushed the need for tritium processing to new levels. These evolving requirements force operations managers and engineers to have a detailed understanding of the capabilities and limitations of the tritium fuel cycle.

Currently, the SRS Tritium Plant relies almost exclusively on high resolution/light isotope mass spectrometers (HRLIMS) for characterization and assurance of process and product quality. In 2001, Mauldin and Gregory outlined the key problems of the aging HRLIMS and associated analytical risks to the tritium plant at SRS. ${ }^{1}$ These HRLIMS are based on 30year old technology and vendors no longer support spectrometer subsystems. As such, spare parts are becoming increasingly difficult to obtain while a commercial-off-the-shelf (COTS) replacement has not been identified. Moreover, many of the necessary analyses identified in the last three decades require accuracy and precision to identify molecules against one another for which HRLIMS is not well suited. These same problematic issues were noted across the DOE in a report from the Network of Senior Scientists and Engineers (NSSE) Advanced Technology Assessment Team (ATAT) that summarized gas sampling and analysis capabilities at each of the DOE sites, the long term viability of HRLIMS, and various technologies being studied to maintain analytical capablilities. ${ }^{2}$

The use of alternative sensing and detection technologies for in-line and real-time analysis would aid process control and optimization and potentially shorten turnaround times as compared to characterization performed by HRLIMS. Although many alternative sensor technologies have been described for analytes of 
interest in tritium fuel cycle processes (Refs. 1-8), none of these technologies have ever been directly implemented or used for detailed chemical analysis of tritiumcontaining isotopes in-situ to the process within the SRS Tritium Plant.

\section{TRITIUM PLANT REQUIREMENTS}

At the Savannah River Tritium Plant, the inability to reach technological adaptation derives primarily on the lack of testing under realistic conditions, which would allow for comprehensive benchmarking and reliability analysis, ultimately providing the necessary confidence for the process engineer to embrace in-line implementation. This testing however, can only be mimicked in a laboratory setting if the researcher has a comprehensive description of all analytes and contaminants present in the targeted system, along with their relative concentrations and ranges. Depending on method, particular contaminant species can have deterring effects on the ability to obtain reliable measurements for a specific analyte at the specific detection requirement and allowed uncertainty. For example, HRLIMS has limited ability to qualify water because of mass interference from ammonia. Ultimately, an assessment providing specific information about analytes of interest including minimum detection requirements, approximate expected concentration ranges, and maximum allowable uncertainty at each desired sampling point is required by the researcher from the process engineer in order to match potential analytical technologies to tritium plant needs.

An initial assessment of the sensing needs of the SRS tritium plant directed attention to at least four specific process subsystems needing detailed gas characterization beyond the current work load of corresponding mass spectrometry analysis (if any). In almost all cases, analytical information requested centered on critical process systems with potentially high degrees of impurities. These subsystems include: A) Thermal Cycling Absorption Process (TCAP) columns where tritium is separated and refined from the process stream and where early detection of inerts and contaminants would aid troubleshooting and potentially significantly reduce the number of samples analyzed by the HRLIMS. B) Tritium Process Stripper (TPS) where the small remainder of advantageous tritium present in the Tritium Plant waste gas must be separated from the waste gas before the waste gas is removed from the facility. C) Diffuser system (DI) stages where hydrogen isotopes are separated from an input mixed gas stream using heated Pd-Ag tubes and where low concentration of impurities present in tritium can rapidly degrade DI stages. And D) Glovebox stripper systems where detection of a long list of potential process impurities could help localize upsets to aid in process troubleshooting, yet HRLIMS does not currently characterize.
A semi-quantitative prioritization process was developed for sensing points across these four process areas. Prioritization of a particular sensing point was directly proportional to the value added by measuring a specific analyte, and inversely proportional to the risks associated with implementing the detection technique at that sensing location. Although each prioritization is specific to a sampling location/point and not meant to provide an overall prioritization analysis against aggregated systems, the risk/benefit analysis serves as a bellwether describing necessity against realistic cost (both direct and indirect) of implementation.

Notable specifics on prioritization results throughout the tritium plant include an increasing demand to qualify and quantify trace levels of impurities including ammonia, water, and methane. Current moisture monitors located in-line are unreliable due to interference from $\mathrm{NH}_{3}$ and $\mathrm{CH}_{4}$. Nor can HRLIMS properly resolve these analytes due to strong overlap of their various isotopologues. Sensing water and volatile hydrocarbons are critical to monitoring Zeolite bed health both in glovebox stripper systems and in-line to tritium plant systems.

\section{POTENTIAL ANALYTICAL METHODS}

Working parallel and complimentary to the sensing requirements assessment was the development of a picklist of alternative technologies available for potential tritium plant implementation at varying levels of readiness. Alternative technologies described in this assessment consisted of both COTS technologies and SRNL-performance tested sensors. SRNL began investigation into alternative technologies in support of the long term strategic plan suggested by Mauldin and Gregory in 2001 and further recommended by US-DOE in 2003 (Ref. 9) to establish potential alternative successors to the HRLIMS for tritium gas analyses. A brief overview of highlights from tritium plant focused sensor technology R\&D at SRS is described in the timeline shown in Figure 1.

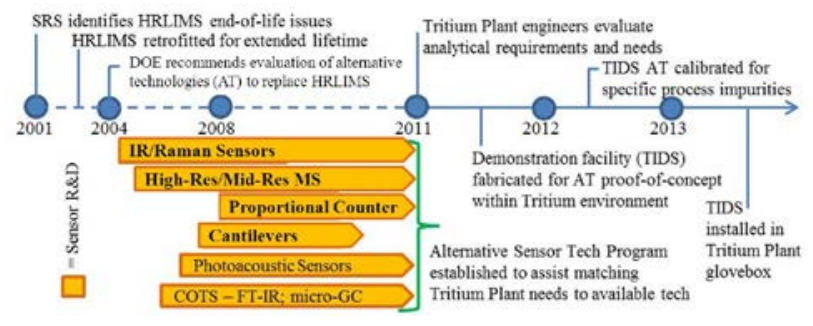

Fig 1. SRS AT R\&D Timeline

While describing every HRLIMS AT SRS has investigated within the past decade is beyond the scope of this paper, it is evident from Figure 1 that numerous 
parallel and competing sensor projects (examples shown in orange arrows) were created from the DOE-wide call for alternative technologies to replace HRLIMS. To prioritize further funding and focus development of these technologies, a comprehensive assessment, written in collaboration with tritium plant engineers, was sent to SRS researchers to gather information on both the technical attributes of each technique as well as compatibility with tritium plant implementation. Information collected included answers to specific questions on ability of alternative technologies to sense/detect tritium plant analytes of interest, requirements to implement a particular instrument in a tritium plant process, present readiness level, and expected timeline/development cost necessary for the technology to reach a state of readiness commiserate with TP deployment.

Gathered information and data on each available alternative sensor technology was used to rank and prioritize sensor technologies against one another based on abilities of specific techniques to satisfy an identified tritium plant need(s). Both SRNL researchers and Savannah River Tritium Plant engineers were given ability to amend the technology pick list if conditions of the plant need changed, if a listed technology reached a higher state of readiness, or if a new sensor was identified. Sensor technologies with the highest level of readiness (e.g. demonstrated by a prototype built or greater) and matching closest to ability to satisfy plant required analysis were suggested for demonstration within the tritium facility.

\section{DEMO FACILITY INSTALLATION}

Evaluating alternative sensor technologies for HRLIMS requires an ability to test new sensors in a process environment. To meet this requirement, a demonstration facility, the Tritium Instrumentation Demonstration Station (TIDS), was fabricated and installed within the tritium plant for purposes of testing SRNL suggested sensors using grab samples taken directly from sampling points previously prioritized by plant engineers. Specific strategy for TIDS was established through creation of a Memorandum of Agreement (MOA) which established roles and responsibilities for both SRNL and the Tritium Plant for design, fabrication, installation, and operation of the TIDS. Derived directly from the MOA, and due to the nature of the facility as a $24 / 7$ operation, it was critical that TIDS installation and operation did not interrupt plant production. To that end, facility space was identified and set aside for TIDS installation, inside a host glovebox not affecting tritium plant production. However, in order to facilitate continuing project progress, all aspects of TIDS development including hazard analysis, safety basis reviews and changes, procedure development, necessary personnel training, and installation were factored into official plant schedules. Although not a process system, TIDS was constructed and installed under all specific Savannah River Tritium Plant safety and operational requirements for equipment in a tritium processing facility. Operation of the TIDS inside the facility safety envelope ensures that any successful alternative technology demonstrated on the TIDS has the maximum chance of transition to in-line deployment.

At present, the TIDS has capability to support and equip up to five separate analytical instruments through an interconnected, tritium compatible manifold. Four of the five total instrumentation areas are currently occupied for demonstration purposes. Instrumentation selected for initial operation has been split between non-massspectrometer COTS units and SRNL-developed Raman to show proof-of-principle of TIDS abilities to chemically analyze process gas and provide immediate benefit to the tritium plant. A 3D model of the TIDS, used to assist SRNL in construction, is shown alongside a frontal image of the facility installed system in Figure 2.

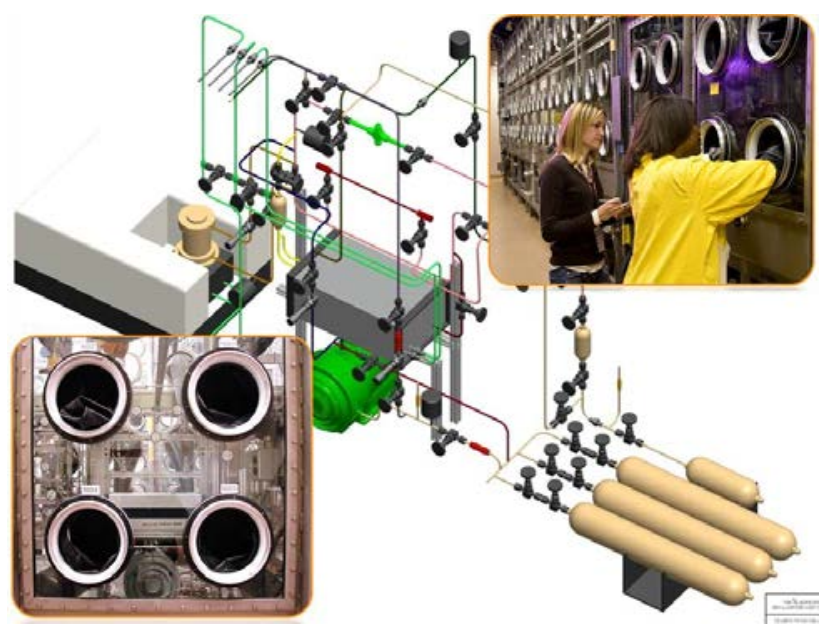

Fig 2. TIDS layout by 3D model. Lower left inset: Front view of installed manifold. Upper right inset: Operation of TIDS inside the TP.

The principle idea is that SRNL available alternative technologies not installed within TIDS are evaluated from the SRNL alternative technology assessment for both maturity level and from the plant assessment for usefulness to tritium plant requirements and needs. This assessment will drive future sensor installation onto TIDS. With agreement between Savannah River Tritium Plant and SRNL, TIDS instrumentation may be amended with merit-driven technologies at any time. This rapid ability to install and evaluate sensor technologies with representative facility process gas provides the plant with on-demand sensor availability during an event, even while the alternative sensor technologies used to analyze 
samples may not be at a maturity level high enough to warrant in-line deployment.

Current TIDS instrumentation includes:

- FT-IR [COTS - Jasco] with 4m multi-pass gas cell

- Micro-GC [COTS - Agilent] with duel 5A molecular-sieve and PlotU columns

- Ion Chamber [COTS - Tyne]; 10cc capacity

- SRNL-developed fiber-optically coupled Raman probe using $532 \mathrm{~nm}$ laser

Analytical abilities of the current instrumentation group match prioritized needs described by tritium plant engineers, specifically for qualitative description of facility process gas and quantitative detection of typical tritium plant gas impurities including $\mathrm{NX}_{3}, \mathrm{CX}_{4}$, and $\mathrm{X}_{2} \mathrm{O}$ where $\mathrm{X}$ is any isotope of hydrogen, along with heavier hydrocarbons and lesser likely impurities including CO and $\mathrm{CO}_{2}$.

\section{CONCLUSIONS}

As HRLIMS nears end-of-life and new processrelated issues are discovered, Savannah River Tritium Plant faces mounting challenges in maintaining the plant's abilities for chemical analysis of process gas. A comprehensive assessment of sensing needs given to SRNL from the Savannah River Tritium Plant provided SRNL researchers the ability to tailor particular alternative sensor technology projects to meet the sensing requirements of specific plant process systems. This semi-quantitative prioritization matrix was used to rank the various analytes in each system, according to process benefit and risk associated with its measurement. Complimentary to the facility assessment, a pick-list of available alternative sensor technologies, both COTS and SRNL-developed (in varying stages of maturity from basic research to finished prototype), was completed by SRNL researchers to evaluate potential sensor technologies against prioritized tritium plant requirements. Alternative sensor technologies at high maturity levels but unproven for in-line plant use can be demonstrated in a tritium plant gas environment using the Tritium Instrumentation Demonstration Station (TIDS), a platform for testing sensors with real process gas. Process gas comes from samples grabbed from various sampling points throughout the facility. The TIDS will be critical to meeting sensing needs described in the Savannah River Tritium Plant and SRNL assessments by providing an established pathway for testing future technologies before full deployment.

\section{ACKNOWLDGEMENTS}

This paper was prepared in connection with work performed under Contract No. DE-AC09-08SR22470 with the U. S. Department of Energy.

\section{REFERENCES}

1. R. LĂSSER and S. GRUNHAGEN, "Gas Chromatography at the Tritium Laboratory Karlsruhe,” ISSN 0947-8620, Karlsruhe: Forschungszentrum Karlsruhe GmbH, (2003).

2. UDA, TATSUHIKO, OKUNO, KENJI, SUZUKI, TAKUMI, NARUSE, YUJI, "Gas Chromatography for measurement of hydrogen isotopes at tritium processing," Journal of Chromatography, 586, (1991).

3. T. OHTA, ET. AL., "HD gas analysis with Gas Chromatography and Quadrupole Mass Spectrometer," Nuclear Instruments Method, 640, (2011).

4. KAWAMURA, ET. AL., "Analysis of hydrogen isotopes with a micro gas chromatograph," Fusion Engineering and Design, 49-50, (2000).

5. M. STRUM, M. SCHLŎSSER, R.J. LEWIS, B. BORNSCHEIN, G. DREXLIN, and H.H. TELLE, "Monitoring of all hydrogen isotopologues at Tritium Laboratory Karlsruhe using Raman spectroscopy,” Laser Physics, 20, (2010).

6. R. LASCOLA, K.E. ZEIGLER, C.S. MCWHORTER, E. VILLA-ALEMAN, L.L. TOVO, and A.E. WARD, "Isotopic hydrogen analysis via conventional and surface-enhanced Raman spectroscopy,” WSRC-MS-2004-00677, Savannah River Site, SC (2004).

7. J. REPPERT, R. LASCOLA, S. MURPH, B. PETERS, S. MCWHORTER, "Advanced Raman Waveguide Sensors for Tritium Processing," FACSS SCIX, Reno, NV, October 2011.

8. L. DONG, J. WRIGHT, B. PETERS, B.A. FERGUSON, F.K. TITTEL, and S. MCWHORTER, "Compact QEPAS sensor for trace methane and ammonia detection in impure hydrogen," Applied Physics B, 107, (2012).

9. G.L. POWELL, NNSE GTIMOG ATAT Gas Sampling and Analysis, YDZ-2436, Y-12 National Security Complex, Oak Ridge, TN (2003). 